\title{
Respostas Cardiovasculares \\ Pós-Exercício de Natação
}

\section{Swimming Post-Exercise Cardiovascular Responses}

Leandro Monteiro Zein Sammour Esteves ${ }^{1}$ Herbert Gustavo Simões ${ }^{1,2}$ Samantha Maria Lemes de Oliveira ${ }^{1}$ Verusca Najara de Carvalho Cunha $a^{1,2}$ João Maurício de Oliveira Coelho ${ }^{1,2}$ Willson Botelho Neto ${ }^{1,2}$ Laila Cândida de Jesus Lima ${ }^{1,2}$ Wesley Salazar de Almeida ${ }^{1,2}$ Carla Britto da Silva $a^{1,2}$

Carmen Sílvia Grubert Campbell ${ }^{1,2}$

1. Universidade Católica de Brasília/UCB

- Taguatinga, Brasília, DF.

2. Programa de Pós-Graduação Strictu Sensu Educação Física - Universidade Católica de Brasília/UCB. Grupo de Estudos do Desempenho Humano e das Respostas Fisiológicas ao Exercício - Brasília, DF.

\section{Endereço para correspondência:} Dra. Carmen Sílvia Grubert Campbell. QS07, LT1 s/n, sala G112, bloco G 72030-170 - Águas Claras, DF, Brasil. E-mail: campbellcsg@gmail.com

\begin{abstract}
RESUMO
Introdução: A crescente procura da natação como um tratamento não-farmacológico da hipertensão arterial tem aumentado. Objetivo: Analisar as respostas da pressão arterial (PA) após o exercício de natação e durante a rotina diária de trabalho de indivíduos pré-hipertensos. Métodos: Oito indivíduos pré-hipertensos $\left(128,2 \pm 7,8 / 77,8 \pm 6,0 \mathrm{mmHg} ; 41,8 \pm 3,2\right.$ anos; $\left.84,7 \pm 17,9 \mathrm{~kg} ; 180,4 \pm 4,8 \mathrm{~cm} ; 27,3 \pm 5,8 \mathrm{~kg} \cdot \mathrm{m}^{2(-1)}\right)$ foram submetidos a duas sessões, sendo uma de natação (SN) e a outra de controle (SC). A PA foi medida no repouso pré-exercício e durante 12 horas de recuperação pós-exercício. Na SN, os pacientes nadaram por $45 \mathrm{~min}$ em uma intensidade moderada a alta (PSE 14,5 $\pm 1,6$ ), e, durante a SC, os sujeitos permaneceram em repouso na posição sentada pelo mesmo período de tempo. Resultados: ANOVA para medidas repetidas evidenciou diminuição significativa $(p \leq 0,05)$ na PAS por duas horas após a SN em relação ao repouso, e uma diferença significativa entre os deltas do PAS ( $\triangle$ - PAS variação na recuperação pós-exercício em relação ao repouso pré-exercício) foi observada entre as sessões na primeira e segunda horas após a recuperação, respectivamente $(\triangle S S=-10,4$ e $-9,3$ vs. $\Delta C S=0,8$ e 4,4mmHg, $p<0,05)$. Não houve diferenças significativas da $P A D$, intra e entre as sessões. Conclusão: $O$ exercício de natação em intensidade moderada a alta foi eficaz para promover redução da PA pós-exercício em indivíduos hipertensos durante a sua rotina de trabalho.
\end{abstract}

Palavras-chave: hipotensão pós-exercício, pressão arterial, frequência cardíaca, percepção subjetiva de esforço.

\begin{abstract}
Introduction: The increasing demand for swimming as a non-pharmacological treatment of hypertension has increased. PURPOSE: To analyze the blood pressure (BP) responses after swimming and during the daily work routine of pre-hypertensive individuals. Methods: Eight male borderline hypertensive individuals (128.2 \pm 7.8 $/ 77.8 \pm 6.0 \mathrm{mmHg} ; 41.8 \pm 3.2$ years; $84.7 \pm 17.9 \mathrm{~kg} ; 180.4 \pm 4.8 \mathrm{~cm} ; 27.3 \pm 5.8 \mathrm{~kg} . \mathrm{m} 2(-1))$ were submitted to a swimming exercise (SS) and control (CS) sessions. BP was measured at pre-exercise rest and during 12 hours of the post-exercise recovery. In SS, patients swan for 45 minutes in a moderate-high intensity (PSE $14.5 \pm$ 1.6), and during CS the subjects remained at seated rest for 45 min. Results: ANOVA for repeated measures evidenced significant decrease $(P \leq 0.05)$ in SBP in the for 2 hours after SS in comparison to rest, and a significant difference between the SBP deltas ( $\triangle$ - SBP variation in the post-exercise recovery compared to pre-exercise rest) was observed when comparing sessions at 1 and 2 hours after recovery, respectively ( $\triangle S S$ $=-10.4$ and -9.3 vs. $\Delta C S=0.8$ and $4.4 \mathrm{mmHg}, \mathrm{p}<0.05$ ). There were not significant differences in DBP within and between sessions. Conclusion: Swimming exercise at moderate-high intensity was effective in promoting post-exercise BP reduction for borderline hypertensive individuals during their daily work routine.
\end{abstract}

Keywords: Post-exercise hypotension, blood pressure, heart rate, perceived exertion

\section{INTRODUÇÃO}

A procura pela prática de exercícios físicos na água tem crescido significativamente nos últimos anos devido ao benefício da redução da sobrecarga sobre as articulações, bem como para promoção de relaxamento muscular pós-exercício ${ }^{(1,2)}$. Além dos objetivos desportivoscompetitivos, a natação também tem sido utilizada com finalidades terapêuticas, como na recuperação de atrofias musculares, no tratamento de problemas respiratórios ${ }^{(3)}$, bem como durante processo de recuperação de lesões ${ }^{(4,5)}$, tornando-se uma forma atrativa de exercício para os idosos e para aqueles com dificuldade de se deslocar por conta do excesso de peso e/ou problemas ortopédicos ${ }^{(6)}$.
Em função de ser realizada na posição horizontal, bem como pelo efeito da pressão hidrostática, a natação promove aumento do retorno venoso e do volume sistólico ${ }^{(7-9)}$, possibilitando, assim, menores valores de frequência cardíaca para manutenção de determinado débito cardíaco durante o exercício. Além disso, devido aos seus benefícios crônicos, a natação foi incluída no quinto relatório do Joint National Committee para detecção, avaliação e tratamento da hipertensão ${ }^{(10,11)}$ como uma modalidade de exercício benéfica para o controle da pressão arterial.

Diversos estudos têm demonstrado que uma única sessão de exercício físico agudo é capaz de reduzir a pressão arterial sistólica (PAS) e diastólica (PAD), no período de recuperação pós-exercício, a valores 
abaixo daqueles aferidos no repouso pré-exercício, ou mesmo daqueles medidos em um dia controle sem exercício ${ }^{(12,13)}$. Este fenômeno é conhecido como hipotensão pós-exercício (HPE), o qual pode ocorrer devido a vários fatores, como maior liberação de substâncias vasodilatadoras $^{(14)}$, redução da atividade nervosa simpática ${ }^{(15)}$ e/ou diminuição do débito cardíaco pós-exercício ${ }^{(16)}$, tendo, portanto, considerável importância clínica.

Porém, pouco se sabe acerca dos efeitos agudos da natação sobre a PA pós-exercício, especialmente em indivíduos pré-hipertensos. Assim sendo, o objetivo do presente estudo foi investigar e comparar as respostas pressóricas após exercício de natação em intensidade moderada a alta por 12 horas pós-exercício de natação e após sessão controle, em indivíduos pré-hipertensos ${ }^{(17)}$.

\section{MATERIAIS E MÉTODOS}

Participaram do estudo oito indivíduos pré-hipertensos, do sexo masculino, trabalhadores em escritório (tabela 1). Todos eram praticantes de natação por, no mínimo, dois anos. Antes do início dos procedimentos, os voluntários assinaram um termo de consentimento livre e esclarecido e foram informados dos riscos, benefícios e possíveis desconfortos advindos de sua participação no estudo, o qual foi aprovado pelo Comitê de Ética em Pesquisa em Seres Humanos da Universidade Católica de Brasília.

Tabela 1. Caracterização da amostra.

\begin{tabular}{l|l}
\hline & Média \pm DP \\
\hline$n$ & 8 \\
\hline Idade (anos) & $41,8 \pm 3,2$ \\
\hline Massa corporal $(\mathrm{kg})$ & $84,7 \pm 17,9$ \\
\hline Estatura (cm) & $180,4 \pm 4,8$ \\
\hline IMC (kg.m2(-1)) & $27,3 \pm 5,8$ \\
\hline PAS rep (mmHg) & $128,2 \pm 7,8$ \\
\hline PAD rep (mmHg) & $77,8 \pm 6,0$ \\
\hline FC rep (bpm) & $62,6 \pm 10,9$ \\
\hline
\end{tabular}

IMC: índice de massa corporal; PAS rep: pressão arterial sistólica de repouso; PAD rep: pressão arterial diastólica de repouso; FC rep: frequência cardíaca de repouso.

\section{Procedimentos}

Os voluntários foram submetidos a duas sessões experimentais: sessão de exercício de natação (SN) e sessão controle sem exercício (SC). As sessões foram realizadas entre 7h30min e 10h30min da manhã, em dias distintos e em ordem randomizada. Os indivíduos foram orientados a não realizar exercício físico e a fazer o mesmo tipo de refeição nas $24 \mathrm{~h}$ anteriores às sessões.

\section{Sessão de exercício de natação (SN)}

Após repouso de 10min na posição sentada, os indivíduos realizaram 45min de natação em intensidade correspondente à percepção subjetiva de esforço (PSE) de 12-14 (moderado a forte) na escala de
Borg de 6-20 pontos ${ }^{(18)}$, sendo que, ao final do exercício, os participantes referiram, em média, PSE de 14,5 $\pm 1,6$. A SN consistiu de cinco minutos de aquecimento nadando à vontade, 30 minutos nadando no estilo crawl, sem pausa, e 10 minutos nadando nos estilos borboleta, costas, peito e crawl sem pausa.

\section{Sessão controle (SC)}

Os procedimentos foram semelhantes aos da SN; entretanto, no período correspondente ao exercício, os indivíduos permaneceram sentados em repouso.

\section{Aferição da PA e da FC}

Em ambas as sessões experimentais, a PA e a FC foram aferidas por meio de equipamento digital (Microlife, Widnau, Suíça) e por frequencímetro Polar (F5, Finlândia) aos cinco e 10 minutos de repouso pré-exercício (considerando-se como valor de repouso (Rep) o resultado da média das duas análises), aos três minutos após o exercício, ainda dentro da piscina na SN (R3min), a cada hora durante as cinco horas seguintes ao exercício ou controle (R1h-R5h) e após 12 horas de recuperação pós-exercício ou controle (R12h). Após a mensuração de R3min, os indivíduos fizeram assepsia na academia durante aproximadamente 15 minutos e seguiram suas rotinas diárias de trabalho em escritório na posição sentada. Antes de todas as mensurações (R1hR12h), os voluntários permaneceram sentados em repouso por pelo menos três minutos e, em seguida, acionaram o aparelho digital para a mensuração da PA.

\section{Análise estatística}

Para comparar os valores de PA intra e entre as sessões experimentais, utilizou-se ANOVA para medidas repetidas, adotando-se $\mathrm{p} \leq$ 0,05 como nível de significância. Todas as análises foram realizadas no programa (Statistica ${ }^{\circledR}$ versão 5.0).

\section{RESULTADOS}

Observou-se HPE de PAS após a SN nos momentos R1h e R2h. Nos momentos R1h, R2h e R3h foram observadas diferenças significativas nos deltas quando comparados à SC, com deltas de variação de pressão arterial (PA pós-sessão menos o valor de PA de repouso) indicando redução significativa pós SN $(-3,5 \mathrm{a}-10,5 \mathrm{mmHg})$, com tendência de aumento pós SC $(0,5$ a $5,5 \mathrm{mmHg})$ (tabela 2$)$.

$\mathrm{Na}$ figura 1 estão apresentados os valores em delta absolutos da PAS, PAD e pressão arterial média - PAM $(\mathrm{mmHg})$ em ambas as sessões experimentais.

Para PAD não foi observada nenhuma alteração significante, nem em relação ao repouso, nem em comparação entre as sessões (tabela 3).

HPE de PAM ocorreu na SN apenas em R1h $(-7,5 \mathrm{mmHg})$; no entanto, foram observadas diferenças entre as sessões nas três primeiras horas de recuperação pós-exercício (R1h-R3h), com maiores quedas na SN (entre $-4,5$ e -7mmHg) em relação ao repouso, enquanto que na SC a PAM, apresentou variação positiva entre 0 e $4 \mathrm{mmHg}$ (tabela 4).

Ao analisar as variações da FC, observou-se que na SN a FC manteve-se significativamente elevada nos momentos imediatamente após o

Tabela 2. Delta de variação da pressão arterial sistólica (PAS) em mmHg.

\begin{tabular}{|c|c|c|c|c|c|c|c|c|c|}
\hline & & Rep & Após 3' & $\mathrm{R} 1 \mathrm{~h}$ & $\mathrm{R} 2 \mathrm{~h}$ & R3h & R4h & R5h & $\mathrm{R} 12 \mathrm{~h}$ \\
\hline \multirow[t]{2}{*}{ SN } & MÉDIA & 128,2 & $-3,6$ & $-10,4^{*} \#$ & $-9,3^{*} \#$ & $-8,6 \#$ & $-1,3$ & $-2,9$ & $-4,2$ \\
\hline & $\mathrm{DP}$ & 7,8 & 6,5 & 3,6 & 6,5 & 4,5 & 8,3 & 5,2 & 10 \\
\hline \multirow[t]{2}{*}{ SC } & MÉDIA & 123,8 & 3,3 & 0,8 & 4,4 & 5,4 & 3,6 & 2,3 & 1,6 \\
\hline & DP & 5,8 & 6 & 6,5 & 8,4 & 7 & 4 & 5,9 & 5,2 \\
\hline
\end{tabular}

${ }^{*} p \leq 0,05$ em relação aos valores de repouso; $\# p \leq 0,05$ em relação ao mesmo momento da SC. 


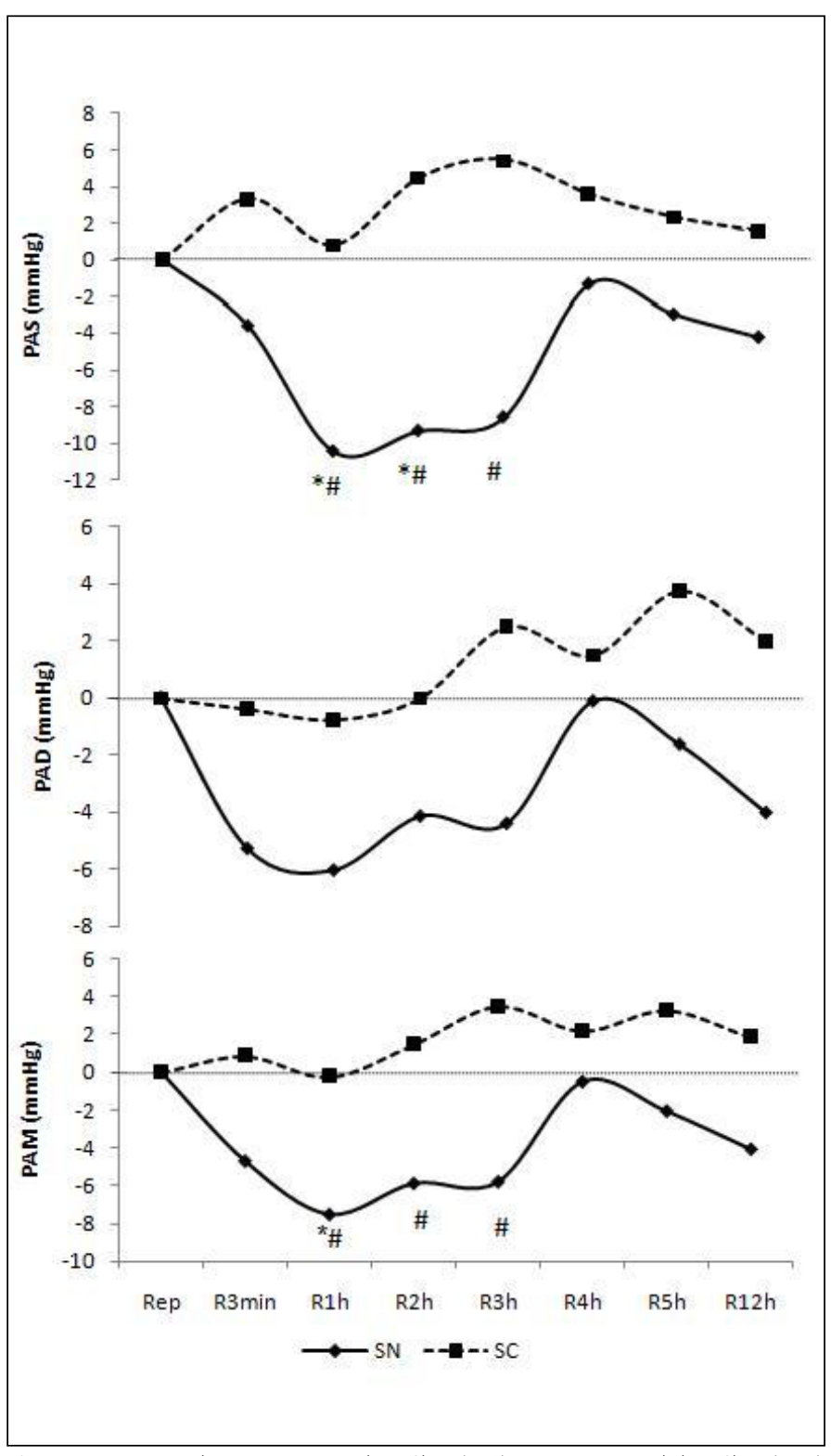

Figura 1. Variação da pressão arterial sistólica (PAS), pressão arterial diastólica (PAD) e pressão arterial média (PAM) durante o repouso (Rep) e a recuperação pós-sessão aos 3min, $1 \mathrm{~h}$ a $5 \mathrm{~h}$ e $12 \mathrm{~h}$ (R1 a R5h e R12h) nas sessões natação (SN) e controle (SC). * $p \leq 0,05$ em relação ao repouso pré-exercício (Rep); \#p $\leq$ 0,05 em relação à sessão controle. exercício $(128,3 \pm 10,9 \mathrm{bpm})$, três minutos após $(84,9 \pm 10,4 \mathrm{bpm})$ e duas horas após $(78,0 \pm 11,3 \mathrm{bpm})$ o término da sessão quando comparadas à $F C$ de repouso $(62,6 \pm 10,9 \mathrm{bpm})$. Não houve variações significativas durante a sessão controle. A FC imediatamente após o exercício (128,3 $\pm 10,9 \mathrm{bpm})$ correspondeu a aproximadamente $70 \%$ da FC máxima $(178,2 \pm 2,2)$ dos voluntários.

\section{DISCUSSÃO}

O presente estudo demonstrou que 45 minutos de exercício de natação foi eficiente em promover significativa queda da PAS pelo período de duas horas e de PAM na primeira hora após o exercício em indivíduos pré-hipertensos do sexo masculino. Ao comparar os deltas de PAS e PAM entre as sessões SN e SC, observou-se que houve significativa diferença nas três primeiras horas pós-exercício, de modo que o exercício resultou em maiores quedas de ambas. Já a queda da PAS e PAM nos momentos R4h, R5h e R12h, foi significativa; porém, os valores de PA sempre permaneceram inferiores aos de repouso, enquanto que, na sessão controle, permaneceram superiores, representando então um benefício importante do ponto de vista clínico promovido pela sessão de natação. Vale ressaltar que os indivíduos mantiveram suas rotinas diárias de trabalho em escritório após o exercício, não ficando em repouso em laboratório, como na maioria dos estudos nessa linha, e ainda assim apresentaram tais reduções nos valores pressóricos, o que aumenta a validade externa dos presentes achados.

Significativa HPE de PAS foi observada por até duas horas de recuperação após o exercício de natação e, além disso, os valores pressóricos pós-exercício se apresentaram menores quando comparados aos da sessão controle. Este efeito hipotensor do exercício de natação pode significar proteção contra eventos cardiovasculares, pois, como sugerido por Whelton et al.(19), uma redução da PAS em $3 \mathrm{mmHg}$ significa uma diminuição de 5-9\% do risco cardiovascular, 8-14\% de infarto e 4\% das causas gerais de morte. No presente estudo, as quedas significativas de PAS ocorreram entre $-8,6$ e $-10,4 \mathrm{mmHg}$ nas três primeiras horas após a SN, enquanto que 12 horas após a SN a PA ainda permanecia inferior ao repouso em $-4,2 \mathrm{mmHg}$ apesar de não ser significativamente diferente. $O$ delta de decaimento da PAS durante as primeiras três horas após o exercício foi aproximadamente três vezes superior ao sugerido por Whelton et al. ${ }^{(19)}$ para a redução dos riscos cardiovasculares dos participantes, enquanto que, após SC, o delta apresentou-se sempre positivo $(0,8$ a $5,4 \mathrm{mmHg})$ demonstrando que, nos dias em que os participantes não realizavam o exercício físico, não tinham esta proteção

Tabela 3. Delta de variação da pressão arterial diastólica (PAD) em mmHg durante o repouso (Rep) e recuperação pós-sessão aos 3min, $1 \mathrm{~h}$ a $5 \mathrm{~h}$ e $12 \mathrm{~h}$ (R1 a R5h e R12h) nas sessões natação (SN) e controle (SC).

\begin{tabular}{c|c|c|c|c|c|c|c|c|c}
\hline & & Rep & Após 3' & R1h & R2h & R3h & R4h & R5h & R12h \\
\hline SN & MÉDIA & 77,8 & $-5,3$ & -6 & $-4,1$ & $-4,4$ & $-0,1$ & $-1,6$ & $-4,0$ \\
\hline & DP & 6 & 3,4 & 5,3 & 5 & 3,7 & 4,1 & 4,9 & 8,5 \\
\hline SC & MÉDIA & 73,9 & $-0,4$ & $-0,8$ & 0 & 2,5 & 1,5 & 3,8 & 2 \\
\hline & DP & 6,7 & 5,8 & 4,9 & 8,5 & 3,5 & 6 & 6,6 & 4,7 \\
\hline
\end{tabular}

Tabela 4. Delta de variação da pressão arterial média (PAM) em mmHg durante o repouso (Rep) e recuperação pós-sessão aos 3min, 1h a 5h e 12h (R1 a R5h e R12h) nas sessões natação (SN) e controle (SC).

\begin{tabular}{c|c|c|c|c|c|c|c|c|c}
\hline & & Rep & Após 3' & R1h & R2h & R3h & R4h & R5h & R12h \\
\hline SN & MÉDIA & 94,6 & $-4,7$ & $-7,5^{*} \#$ & $-5,9 \#$ & $-5,8 \#$ & $-0,5$ & $-2,1$ & $-4,1$ \\
\hline & DP & 6,3 & 3,4 & 4,5 & 4,3 & 3,6 & 4,9 & 4,5 & 8,3 \\
\hline SC & MÉDIA & 90,5 & 0,9 & $-0,2$ & 1,5 & 3,5 & 2,2 & 3,3 & 1,9 \\
\hline & DP & 6,1 & 4,7 & 2,3 & 4,7 & 2,3 & 3,8 & 4,7 & 3,2 \\
\hline
\end{tabular}

${ }^{*} \mathrm{p} \leq 0,05$ em relação ao repouso; \#p $\leq 0,05$ em relação ao mesmo momento da SC. 
de redução de riscos cardiovasculares. Supõe-se que a redução da PAS ainda poderia ser maior caso o indivíduo permanecesse em repouso no ambiente de laboratório.

Resultados similares foram observados por MacDonald et al. ${ }^{(20)}$ ao avaliarem indivíduos pré-hipertensos durante atividades simuladas da vida diária (ASVD) após prática de exercício físico. Os autores observaram que, nos primeiros 70 minutos após o exercício, houve quedas significativas da PAS, PAD e PAM (-16, -5 e $-8 \mathrm{mmHg}$, respectivamente) quando comparados ao dia que os voluntários não realizaram exercício físico previamente as ASVD.

Estes achados estão de acordo com outros estudos que demonstraram HPE tanto de PAS como de PAD $(-2 \pm 2$ e $-17 \pm 2 \mathrm{mmHg})$ em indivíduos normotensos e hipertensos ${ }^{(21,22)} \mathrm{em}$ diferentes modalidades como esteira, musculação e ergômetro de braço e entre outras modalidades em intensidades relativas à máxima ou abaixo e acima do limiar de lactato ${ }^{(13,16,23,24)}$, sendo que o exercício aeróbio mais intenso ${ }^{(25,}$ 26), mais prolongado(12) e que envolve grandes grupos musculares ${ }^{(27)}$ mostrou-se como a modalidade de exercício que promoveu quedas mais acentuadas na PA.

Porém, para que o exercício tenha importância clínica sobre as respostas da PA, é necessário que esta perdure por várias horas após o exercício físico ${ }^{(12,15)}$.
O protocolo de exercício utilizado no presente estudo simulou uma aula típica de natação, com duração média de 45 minutos e PSE em torno de 14 pontos na escala de Borg de 6-20 pontos (entre pouco cansativo e cansativo), e correspondeu a 71,8 $\pm 6,6 \% \mathrm{FC}_{\max }$ de acordo com a fórmula de Tanaka et al.(28), o que está próximo das intensidades dos exercícios da literatura consultada.

Tal exercício foi suficiente para que ocorresse HPE nas primeiras horas após o exercício, bem como menores valores de PAS e PAM que o dia controle sem exercício, durante a rotina normal de trabalho dos participantes em escritório. Assim, conclui-se que 45 minutos de natação com intensidade moderada-alta (classificada como ligeiramente cansativa pela escala de percepção subjetiva de esforço de Borg) promoveu HPE de PAS e PAM e resultou em valores pressóricos abaixo daqueles observados em um dia sem exercício, durante a rotina diária de trabalho, reforçando os benefícios da natação no tratamento não farmacológico e prevenção da HAS.

Todos os autores declararam não haver qualquer potencial conflito de interesses referente a este artigo.

\section{REFERÊNCIAS}

1. Harrison RA, Hillman M, Bulstrode S. Loading of the lower limb when walking partially immersed: implications for clinical practice. Physiotherapy. 1992;78:164-6.

2. Gabilan YPL, Perracini MR, Munhoz MSL, Ganança FF. Fisioterapia aquática para reabilitação vestibular. ACTA ORL. 2006;25-30.

3. Arandelović M, Stanković I, Nikolić M. Swimming and persons with mild persistent asthma. Scientific World Journal. 2007;17:1182-8.

4. Douglas JF, Gasiorek JM, Swaffield JA. Fluid mechanics. Londres: Pitman, 1979.

5. Dundar U, Solak O, Yigit I, Evcik D, Kavuncu V. Clinical effectiveness of aquatic exercise to treat chronic low back pain: a randomized controlled trial. Spine. 2009;34:1436-40.

6. Floras JS, Notarius CF, Harvey PJ. Exercise training - not a class effect: blood pressure more buoyant after swimming than walking. J Hypertens. 2006;24:269-72.

7. Avellini BA, Shapiro Y, Pandolf KB. Cardio-respiratory physical training in water and on land. Eur J Appl Physiol Occup Physiol. 1983;50:255-63.

8. Christie JL, Sheldahl LM, Tristani FE, Wann LS, Sagar KB, Levandoski SG, et al. Cardiovascular regulation during head-out water immersion exercise. J Appl Physiol. 1990;69:657-64.

9. Sheldahl LM, Wann LS, Clifford PS, Tristani FE, Wolf LG, Kalbfleisch JH. Effect of central hypervolemia on cardiac performance during exercise. J Appl Physiol. 1984;57:1662-7.

10. Guidelines Subcommittee: 1993 Guidelines for the Management of Mild Hypertension: Memorandum from a World Health Organization/ International Society of Hypertension Meeting. J Hypertens. 1993;11:905-18

11. The fifth report of the Joint National Committee on Detection, Evaluation and Treatment of High Blood Pressure (JNC V). Arch Intern Med. 1993;153:154-83.

12. Forjaz CLM, Santaella DF, Rezende LO, Barretto ACP, Negrão CE. A duração do exercício determina a magnitude e a duração da hipotensão pós-exercício. Arq Bras Cardiol. 1998;70:99-104.

13. Lima LC, Assis GV, Hiyane W, Almeida WS, Arsa G, Baldissera V, et al. Hypotensive effects of exercise performed around anaerobic threshold in type 2 diabetic patients. Diabetes Res Clin Pract. 2008;81:216-22

14. Moraes MR, Bacurau RPF, Ramalho JDS, Reis FC, Casarini DE, Chagas JR, et al. Increase in kinins on postexercise hypotension in normotensive and hypertensive volunteers. Biol Chem. 2007;388:533-40.

15. Kenney MJ, Seals DR. Postexercise Hypotencion: Key Features, Mechanisms, and Clinical Significance. Hypertension. 1993;22:653-64
16. Rezk CC, Marrache RCB, Tinucci T, Mion DJr, Forjaz CL. Post-resistance exercise hypotension, hemodynamics, and heart rate variability, influence of exercise intensity. Eur J Appl Physiol. 2006;98:105-12.

17. Chobanian A, Bakris G, Black H, Cushman W, Green L, Izzo JJ, et al. Seventh report of the Joint National Committee on Prevention, Detection, Evaluation, and Treatment of High Blood Pressure. Hypertension. 2003;42:1206-52.

18. Borg G. Perceived exertion and pain scales. Champain: Human Kinetics, 1998

19. Whelton PK, He J, Appel L, Cutler JA, Havas S, Kotchen TA, et al. Primary prevention of hypertension: clinical and public health advisory from the national high blood pressure education program. JAMA 2002;288:1882-8.

20. MacDonald JR, Hogben CD, Tarnopolsky MA, MacDougall JD. Post exercise hypotension is sustained during subsequent bouts of mild exercise and simulated activities of daily living. J Hum Hypertens. 2001;15:567-71.

21. Brandao RMU, Alves MJ, Braga AM, Teixeira OT, Barretto AC, Krieger EM, et al. Postexercise blood pressure reduction in elderly hypertensive patients. J Am Coll Cardiol. 2002;39:676-82.

22. Santaella DF, Campos RN, Araujo EA, Ortega K, Tinucci T, Itiki C, et al. Exercício e relaxamento alteram a modulação autonômica da hipotensão pós-exercício diferentemente em normotensos e hipertensos. Hipertensão. 2003;6(Supl):36.

23. Lizardo JHF, Modesto LK, Campbell CSG, Simões HG. Hipotensão pós exercício: comparação entre diferentes intensidades de exercício em esteira ergométrica e cicloergômetro. Rev Bras Cineatropom Desempenho Hum. 2007;9:115-20.

24. Cunha GA, Rios ACS, Moreno JR, Braga PL, Campbell CSG; Simões HG, et al. Hipotensão pós exercício em hipertensos submetidos ao exercício aeróbio de intensidades variadas e exercício de intensidade constante. Rev Bras Med Esporte. 2006;12:313-7.

25. Quinn TJ. Twenty-four hour, ambulatory blood pressure responses following acute exercise: impact of exercise intensity. J Hum Hypertens. 2000;14:547-53.

26. Forjaz CLM, Cardoso CGJr, Rezk CC, Santaella DF, Tinucci T. Postexercise hypotension and hemodynamics: the role of exercise intensity. J Sports Med Phys Fitness. 2004;44:54-62.

27. MacDonald JR, MacDougall JD, Hogben CD. The effects of exercising muscle mass on post exercise hypotension. J Hum Hypertens. 2000;14:317-20

28. Tanaka H, Monahan KD, Seals DR. Age-predicted maximal heart rate revisited. J Am Coll Cardiol. 2001;37:153-6 